\title{
Lebensqualität von Brustkrebspatientinnen wåhrend der Misteltherapie: Eine qualitative Studie
}

\author{
Mathias Brandenberger ${ }^{a, b} \quad$ Ana Paula Simões-Wüst ${ }^{a}$ \\ Matthias Rostock ${ }^{c} \quad$ Lukas Rist $^{\mathrm{a}} \quad$ Reinhard Saller $^{\mathrm{c}}$ \\ ${ }^{a}$ Forschungsabteilung, Paracelsus-Spital Richterswil, Schweiz \\ bPsychosomatische Klinik Sonneneck, Badenweiler, Deutschland \\ Institut für Naturheilkunde, UniversitätsSpital Zürich, Schweiz
}

\section{Schlüsselwörter}

Krebs · Lebensqualität · Mistel · Qualitative Forschung · Interviews . Inhaltsanalyse

\section{Zusammenfassung}

Hintergrund: Obwohl mehrere klinische Studien gezeigt haben, dass Misteltherapie (MT) die Lebensqualität von KrebspatientInnen verbessern kann, liegen qualitative Informationen über die Art der Verbesserungen bis heute nur sehr begrenzt vor. Patienten und Methoden: Diese explorative, prospektive Kohortenstudie zur Untersuchung der Lebensqualität unter MT bei KrebspatientInnen ( $n=25$, ambulantes Setting, Therapieschema mit s.c.-Applikation) mit polymodalem Therapiekonzept wurde von der Ethikkommission des Kantons Zürich, Schweiz, bewilligt. Die Erfassung der Daten erfolgte zu Beginn der MT und nach 3 Monaten - einerseits mittels Fragebogen (EORTC QLQC30 Version 3.0, $n=21$ ), während anderseits ein Teil der PatientInnen $(n=17)$ für ein Interview gewonnen werden konnte. Die Interviews wurden einer qualitativen Inhaltsanalyse $(n=12)$ unterzogen. Für die vorliegende Arbeit wurden die Texte der Interviews der 4 Brustkrebspatientinnen extrahiert und analysiert. Ergebnisse: Es zeigte sich, dass die Patientinnen im untersuchten Zeitraum generell und/oder punktuell eine subjektiv höhere Lebensqualität generieren konnten. Dazu beigetragen haben Verbesserungen des Befindens, aber insbesondere auch der Erwerb von persönlichen Fähigkeiten, die im Zusammenhang mit einer gelungenen, bedürfnisorientierten Adaptation im psychosozialen Umfeld angesehen werden können. Hinsichtlich der subjektiv gewonnenen Lebensqualität - im Sinn einer individuell sinnrichtigen und stimmigen therapeutischen Strategie - traten bei den einzelnen Patientinnen und in Bezug auf die MT wesentlich relevante Ansprüche an das Setting und die Art der therapeutischen Begleitung zutage. Häufig waren sowohl die Krebserkrankung als auch die Therapien mit einer subjektiven Bedeutung belegt, die (psychodynamisch) bewältigungsrelevant sein kann. Schlussfolgerungen: Eine Krankheitsbewältigung erscheint insofern entsprechend der jeweiligen Bedürfnisse erfolgreich, wenn die Behandlung in diesem Sinn ganzheitlich angelegt ist.

\section{Keywords}

Cancer - Quality of life - Mistletoe - Qualitative research · Interviews . Content analysis

\section{Summary}

Quality of Life of Breast Cancer Patients during Mistletoe Therapy:

A Qualitative Study

Background: Although several clinical studies have shown that mistletoe therapy (MT) may improve patients' quality of life, qualitative information on the nature of these improvements is still scarce. Patients and Methods: This exploratory, prospective cohort study was authorised by the ethical commission of canton Zurich, Switzerland, and comprised 25 patients with different types of cancer, which were surveyed during subcutaneous MT as part of a multimodal treatment. Patients filled in the EORTC QLQ-C30 Version 3.0 questionnaire at the beginning of MT ( $n=25)$ and 3 months later $(n=21)$. Given the patients' additional agreement, they were interviewed at both occasions $(n=17)$, and the interviews were submitted to a qualitative content analysis $(n=12)$. Among these, there were 4 patients with breast cancer, whose interview texts have been extracted and analysed in this manuscript. Results: The data show that after 3 months of MT the patients perceived general or specific improvements of their quality of life. These improvements comprised not only an increased well-being but also the acquirement of personal abilities, which can be seen as associated with successful and necessities-related adaptations in the psychosocial environment. In the context of MT, the patients revealed their requirements concerning the setting and the type of therapeutic supervision, which might reflect a subjective improvement of quality of life. Both cancer disease and therapies were often associated with subjective meanings, which might be important for (psychodynamic) coping. Conclusion: With respect to the patients' requirements, coping with the disease appears to be successful if the treatment is holistically applied.

\section{KARGER \\ Fax +497614520714 Information@Karger.de} www.karger.com
Dr. Ana Paula Simões-Wüst

Forschungsabteilung, Paracelsus-Spital Bergstrasse 16, 8805 Richterswil, Schweiz simoes@paracelsus-spital.ch 


\section{Mots-clés}

Cancer · Qualité de vie · Gui · Recherche qualitative · Entretiens .

Analyse du contenu

\section{Résumé}

Qualité de vie des patientes présentant un cancer du sein au cours de leur traitement par le gui: étude qualitative

Contexte: De nombreuses études ont montré que le traitement par le gui peut améliorer la qualité de vie de patientes présentant un cancer du sein. Cependant, les informations qualitatives quant au type d'améliorations qu'il engendre restent aujourd'hui très limitées. Patients et méthodes: Cette étude de cohorte prospective et exploratoire destinée à l'analyse de la qualité de vie au cours d'un traitement par le gui chez des patientes atteintes d'un cancer ( $n=25$, cadre ambulatoire, schéma thérapeutique en application sous-cutanée) avec concept thérapeutique polymodal a été approuvée par la commission d'éthique du canton de Zurich, Suisse. La collecte des données a été effectuée au début du traitement et après 3 mois, d'une part à I'aide de questionnaires (EORTC QLQ-C30 Version 3.0, $\mathrm{n}=21$ ) et d'autre part par des entretiens menés auprès d'une partie des patientes $(n=17)$. Les entretiens ont été soumis à une analyse de contenu qualitative $(n=12)$. Les textes des entretiens de 4 patientes atteintes d'un cancer du sein ont été extraits et analysés pour les besoins du présent travail. Résultats: Cette étude a montré que les patientes ont pu améliorer leur qualité de vie subjective au cours de la période d'étude de manière générale ou ponctuelle. Ont contribué à cela les améliorations du bien-être, et plus encore le développement de facultés personnelles qui peuvent être considérées en lien avec l'adaptation réussie et orientée sur les besoins dans l'environnement psychosocial. En ce qui concerne la qualité de vie subjective acquise, en tant que stratégie thérapeutique individuelle cohérente et sensée, les patientes ont exprimé, suite au traitement par le gui, des exigences tout à fait pertinentes relatives au cadre et au type d'accompagnement thérapeutique. Elles ont souvent donné au cancer ainsi qu'à son traitement un sens subjectif, qui peut s'avérer efficace dans la lutte contre la maladie (approche psychodynamique). Conclusions: La lutte contre la maladie en fonction des différents besoins semble donc réussir quand le traitement est dicté par une approche holistique.

\section{Einleitung}

Präparate aus Viscum album (Mistel) gehören zu den am meisten eingesetzten Behandlungsformen komplementärer Krebstherapie in Europa [1]. Die Mistelpräparate kommen dabei oft zusammen mit einer konventionellen Basistherapie bzw. als Zusatztherapie zum Einsatz [2]. Effekte therapeutischer Wirksamkeit wurden in mehreren klinischen Studien aufgezeigt und sind in einigen kürzlich erschienenen Übersichtsarbeiten beschrieben worden [1,
3-6]. Während widersprüchliche Resultate zum möglichen Nutzen hinsichtlich einer Verbesserung der Lebenserwartung sowie der Tumorregression durch eine Misteltherapie (MT) bestehen, steigt die Evidenz eines positiven Effekts auf die Lebensqualität (Quality of Life, QoL) [6, 7]. Anzumerken gilt es diesbezüglich, dass noch wenig qualitative Daten zur Art der Lebensqualitätsverbesserungen vorliegen.

In klinischen Studien wird die QoL in der Regel mittels standardisierter Fragebögen erhoben. So ist es möglich, die QoL für eine Vielzahl von PatientInnen objektivierend zu quantifizieren. Anderseits mangelt es somit an der Erfassung von detaillierteren subjektiven Dimensionen der QoL - insbesondere die persönlichen Bewältigungsstrategien (Anpassung an eine existenzielle Bedrohung) betreffend. Dieser Mangel veranlasste uns zur Durchführung der vorliegenden Studie, die kürzlich in einer englischsprachigen Peer-reviewed-Fachzeitschrift veröffentlicht wurde [8]. Für die vorliegende Fassung haben wir die Daten der von dieser Krankheit betroffenen Patientinnen extrahiert und aufbereitet.

\section{Patienten und Methoden}

Diese prospektive Vorher/Nachher-Kohortenstudie fand am ParacelsusSpital in Richterswil, Schweiz, statt. Hierbei wurden fortlaufend PatientInnen $(n=25)$ in die laufende Untersuchung mit einbezogen, die aufgrund verschiedener Krebserkrankungen in eine ambulante Behandlung mittels subkutaner Applikation eines anthroposophischen Mistelpräparats eintraten. Es bestanden keine Ausschlusskriterien. Die PatientInnen waren insbesondere hinsichtlich der Wahl ihrer Therapien nicht limitiert. Zur Anwendung kamen folgende Mistelpräparate, die zwei- bis dreimal pro Woche injiziert wurden: Iscador ${ }^{\circledR}(\mathrm{n}=13$; Qu, M oder P; Weleda AG, Arlesheim, Schweiz), Abnoba Viscum ${ }^{\circledR}(\mathrm{n}=11$; Qu oder A; Abnoba GmbH, Pforzheim, Deutschland) und Isorel ${ }^{\circledR}(\mathrm{n}=1$; Novipharm $\mathrm{GmbH}$, Pörtschach am Wörthersee, Österreich).

Die Studie wurde von der Ethikkommission des Kantons Zürich genehmigt und entsprechend der Deklaration von Helsinki durchgeführt. Alle an der Studie teilnehmenden PatientInnen unterzeichneten am Anfang der Studie eine Einwilligungserklärung. Sie füllten sodann verschiedene Fragebogen zur QoL aus - unter anderem den EORTC QLQ-C30 Version 3.0, und zwar zu Beginn der Behandlung sowie 3 Monate später. Auch ein Fragebogen zur MT wurde zu beiden Zeitpunkten ausgefüllt. Die Studie beinhaltete eine Inhaltsanalyse der halbstrukturiert geführten Interviews (offenes Ende). Die Interviews wurden in deutscher Sprache (so weit möglich in dem lokalen Dialekt) durchgeführt. Die Abschriften der Interviews wurden sodann einer Inhaltsanalyse unterworfen [9]. Diese bestand aus den folgenden Schritten: a) der Interviewtext wurde mehrere Male gelesen, b) der Text wurde auf Sätze untersucht, die Informationen über die a priori definierten Themen beinhalteten, c) die entsprechenden Sätze wurden markiert, d) neue Themen (die während des Lesens entdeckt worden sind) wurden dem Procedere - wie unter b) und c) beschrieben unterworfen, e) die ausgesonderten Informationen (markierte Sätze) wurden sodann sequenziell komprimiert, tabellarisch gefasst und geordnet, und f) es wurden wesentliche Zitate für Darstellungszwecke extrahiert. Darüber hinaus wurden nachträglich wahrgenommene Themen in der Analyse mit berücksichtigt [10]. Die PatientInnen wurden mit einem Code erfasst. Dieser beinhaltete das Geschlecht und das Alter: F45 beispielsweise steht für eine 45-jährige weibliche Patientin. 


\section{Ergebnisse}

Die mit dem EORTC QLQ-C30 zu Beginn der Behandlung und 3 Monate später $(n=21)$ gewonnenen Daten zeigten Verbesserungen in einigen wichtigen Aspekten der QoL, zum Teil auf statistischem Signifikanzniveau. Die Ergebnisse früherer Studien fanden dabei Bestätigung; die weiteren verwendeten Fragebogen (SELT-M, Herdecker) zeigten Resultate mit entsprechender Tendenz, allerdings weniger eindrücklich [11]. Die quantitativen Ergebnisse dienten in erster Linie zur Vergleichbarkeit des qualitativen Teils dieser Studie mit früheren Arbeiten. Dies gilt auch für die hier ebenfalls nicht gezeigten soziodemographischen Daten. Die vorliegende Darstellung der Daten beschränkt sich dabei auf Resultate der qualitativen Inhaltsanalyse der Interviews mit den Brustkrebspatientinnen. So kann aus unserer Sicht am besten aufgezeigt werden, was eine Verbesserung der QoL für einzelne BrustkrebspatientInnen unter Misteltherapie konkret bedeuten kann (für eine vollständige Darstellung siehe [8]).

\section{Zusätzliche Therapien und hilfreiche Aktivitäten}

Die konventionellen Therapien der in dieser Analyse berücksichtigten Patientinnen sind in Tabelle 1 abgebildet. Dort wird auch die Zeit seit dem Zeitpunkt der Diagnosestellung angegeben. Auffallend ist, dass alle 4 Brustkrebspatientinnen vor der MT eine chirurgische Operation hatten. Auch noch vor der MT wurde eine angebotene Chemotherapie von einer Patientin abgelehnt; eine Radiotherapie wiederum wurde von 2 Patientinnen abgelehnt. Alle 4 Patientinnen wiesen eine Antihormonbehandlung auf; eine Patientin nahm Bisphosphonate aufgrund von Knochenmetastasen. 3 Patientinnen setzten die MT als eine ergänzende Therapie zur primär gesetzten konventionellen Behandlungsmethode (Chemotherapie, Radiotherapie, Operation) ein, vornehmlich mit einem sekundären, unterstützenden Charakter. Nur bei der Patientin F56 war die MT eine, wenn nicht die wesentliche Behandlung (Tab. 1); sie entschied sich sowohl gegen eine Chemoals auch eine Strahlentherapie, unterzog sich aber einer partiellen Operation und konnte sich auch für eine Antihormontherapie entscheiden. Diese Patientin war sodann im befragten Kollektiv die einzige Patientin, die nicht grundsätzlich eine konventionelle Therapie zentral favorisierte. Bei dieser Patientin wurde die MT im Rahmen einer komplementärmedizinischen Therapiestrategie bei wei- terhin stark psychotherapeutischer Orientierung durchgeführt und zeigte sich als intensiver Versuch, die therapeutische Strategie mit dem subjektiven Krankheitsmodell (Krebs als Ausdruck eines Traumas, bewusste Selbstfürsorge) in einen logischen Zusammenhang zu bringen. Auch bei den anderen Patientinnen zeigten sich individuelle «strategierationale» Gründe für die Wahl der MT und anderer zusätzlicher Therapien, auf die an anderer Stelle noch vertieft eingegangen werden soll.

Die Befragungen verdeutlichten insgesamt eine weite Aufstellung von nichtkonventionellen Therapien sowie bewältigungswirksamen Aktivitäten - hierbei fiel insbesondere auch auf, dass die PatientInnen in den Interviews deutlich ausführlichere Rückmeldungen zu zusätzlichen Therapien machten als anlässlich der entsprechend konkreten Frage in den Fragebogen zur MT (siehe [8]). Die Brustkrebspatientinnen erwähnten die Eurythmie (F56, gegen Depressionen), die Familienaufstellung (F56, psychotherapeutische Klärung und Bearbeitung eines Kindheitstraumas), Lymphdrainage (F56, Verbesserung des Zustandes nach Operation), Akupunktur (F56, gegen Schmerzen) und Psychotherapie (F42, im Sinne einer allgemeinen unterstützenden Orientierung). F42 reaktivierte und intensivierte zudem eine "therapeutisch» wirksame Pferdebeziehung, was mit Kraft- und Mutzuwachs, verbesserter Funktionalität und Belastbarkeit einherging. 2 Patientinnen (F63, F69) machten formal zwar keinen Gebrauch von anderen komplementärmedizinischen Therapien, jedoch ergaben sich in den Interviews interessante und erheblich «therapierelevante» Hinweise. So erwähnte F69, dass ihr ein befreundetes Ehepaar «positives Denken» beigebracht habe.

\section{Bedeutung der Erkrankung für die Patientinnen und ihre Bewältigung}

Die Brustkrebspatientinnen sahen die Erkrankung als eine Chance oder einen Hinweis, etwas in ihrem Leben zu verbessern, häufig im Sinne einer mehr bedürfnisorientierten Abgrenzung (F42: «Ich probiere, mich nicht mehr hetzen $\mathrm{zu}$ lassen. Ich probiere, eines nach dem anderen $\mathrm{zu}$ machen.»). Sodann wurde ein Impuls angesprochen, um subjektiv-ätiologisch relevante, vergangene Ereignisse aufzuarbeiten (F56: «Das sind so subtile Sachen, aus der Geschichte, was im Kern in der Kindheit passiert ist ... erst jetzt ... wird sich das ... diese Energien, wie befreien ... dass ich zu meiner Wahrheit komme ... zu dem, was ich

Tab. 1. Zusätzliche konventionelle Therapien, die die Brustkrebspatientinnen durchführten

\begin{tabular}{llllllllc}
\hline Patientin & OP & $\begin{array}{l}\text { Chemotherapie } \\
\text { vorher }\end{array}$ & $\begin{array}{l}\text { Chemotherapie } \\
\text { während MT }\end{array}$ & $\begin{array}{l}\text { Radiotherapie } \\
\text { vorher }\end{array}$ & $\begin{array}{l}\text { Radiotherapie } \\
\text { während MT }\end{array}$ & Hormontherapie & Bisphosphonate & Zeit seit Diagnose \\
\hline F56 & X & abgelehnt & - & abgelehnt & - & X & - & Wochen \\
F63 & X & - & - & abgelehnt & - & X & Wochen \\
F69 & X & - & - & X & X & X & X & 6 Monate \\
F42 & X & X & - & - & X & - & 11 Monate \\
\hline
\end{tabular}


bin.»). Darüber hinaus wurde die Erkrankung als ein «Lehrer» aufgefasst (F56: «... der mir jeden Moment zeige, was mit mir vorgehe»; Anmerkung: im Sinn von «bewusster zu machen»). Bezüglich einer möglichen Bedeutung der Erkrankung liessen sich bei 3 Patientinnen im Rahmen der Interviews subjektive Ursache-Wirkungs-Zusammenhänge ausmachen, mit direkter biografischer Kausalitätsauffassung (F56) sowie vermuteten Zusammenhängen subjektiv unklarer, aber klar ängstigender (F63) oder mehr bewältigungsorientierter Wertigkeit (F42). Diese Wertigkeit schien die Triggerung einer selbstreflektierenden bedürfnisorientierten Autonomisierung («Individuation») zu sein, sowohl beruflich als auch in den nahen Beziehungen.

\section{Patientinnenbeurteilung der Misteltherapie}

Im Zusammenhang mit der MT wurde längsschnittlich von den Patientinnen berichtet: weniger Depression (F63: (MT ist für mich) «sehr gut und ich könnte es jedem empfehlen ... (der stärkste Effekt) war gemütsmässig ... Ich hatte Neigung gehabt zu leichten Depressionen und ... (jetzt) kann ich das (Anmerkung: Belastungen, insbesondere Konflikte mit der Mutter) viel gelassener hinnehmen»), verbesserte emotionale Stabilität (F63: «nicht alles auf mich so tragisch wirken lassen ... Hier vermute ich doch sehr stark, dass das mit einem Einfluss von der Mistelkur in Verbindung ist»; F42: "... ich bin etwas konstanter in der Stimmung») und sich kräftiger fühlen (F56: «Ich spüre mich etwas kräftiger innerlich ... stärker innerlich»). F69 hat sodann im Zusammenhang mit der MT geäussert, dass sie diese Behandlung "wider» den Arzt aufgenommen habe, was als Autonomieschritt verstanden werden kann. In ihren Äusserungen im Interview ist diesbezüglich ein bewältigungswirksamer Impuls auszumachen, der eine Rolle gespielt haben dürfte anlässlich des Coping mit Verlaufsbefunden sowie einer geplanten Reise nach Übersee zum Besuch ihrer Tochter. Eine Patientin äusserte sodann rückläufige Arthroseschmerzen (F63: «Das bringt man nicht mehr weg, aber die Schmerzen sind nicht mehr so gross ... Ich kann auch zum Teil wieder handarbeiten.»). Diese Patientin (F63) sprach ein differenziertes Stufenschema in Bezug auf ihre chronische Arthritis an; dementsprechend würden zunächst komplementäre Therapien zur Anwendung kommen - und wenn es nicht mehr anders geht die konventionellen Behandlungen. Anlässlich sehr differenzierter Rückmeldungen zur Wirkung/Nebenwirkung der MT zeigte sich eine sehr bewusst geführte Auseinandersetzung mit verschiedenen therapeutischen Optionen.

Analysen der (zweiten) Interviews zeigten auf, dass es in manchen Fällen zu Reaktionen unter MT kam, die einerseits als Nebenwirkungen gedeutet werden können, anderseits als Bestandteil von erwarteten Reaktionen der subkutanen MT aufzufassen sind: Rötungen wurden von zwei Brustkrebspatientinnen (F69 und F42) beschrieben.
Tab. 2. Zusammenfassung der Inhaltsanalyse der Interviews

\begin{tabular}{ll}
\hline Parameter & Patientinnen \\
\hline $\begin{array}{l}\text { Wahrgenommene Veränderungen } \\
\text { Stärkere Vitalität }\end{array}$ & F56, F63, F69 \\
Grössere Autonomie & F63 \\
Bessere Akzeptanz der Krankheit & F56 \\
Berichtete Leistungen & \\
Änderungen im Berufsleben & F42 \\
Neue Projekte & F69 \\
Gefühl der Befreiung & F56, F42 \\
Kapazität, eigene Entscheidungen & F56, F63 \\
zu treffen oder Prioritäten zu etablieren & \\
\hline
\end{tabular}

Keine der Patientinnen unterbrach die MT aufgrund der Reaktionen.

Von den Patientinnen berichtete persönliche Erfolge und Leistungen

Wie in Tabelle 2 zusammengefasst, erwähnten manche Patientinnen nach 3 Monaten MT bzw. im Rahmen des zweiten Interviews eine neue Art von Akzeptanz / einen adaptierten Umgang mit ihrer Erkrankung / eine regenerative Kräftigung, Vitalisierung (F56: «Ich merke jetzt, dass ich viel näher bin zu meiner Krankheit»; F56: «Ich spüre mich etwas kräftiger innerlich»; F63: «Ich bin im Moment überzeugt, dass ich gesund bin» sowie «Manchmal denke ich mir, ich fühle mich überhaupt gesund ... für was mache ich das alles, es geht mir so gut ...»). Andere Patientinnen brachten ihre Verbesserungen in einen Zusammenhang mit mehr Autonomie (F63: «Ich grenze mehr Sachen $a b$... ich nehme vielleicht gewisse Dinge ernster ... befasse mich mit diesen Dingen mehr und grenze vieles aus, oder manches ... ich lasse nicht mehr alles so nah an mich heran kommen, ich schiebe ein bisschen weg ... ich sortiere aus, oder sondiere ein bisschen mehr ... ich fühle mich ein bisschen sicherer»). Mitunter wurden von den Patientinnen neue Leistungen berichtet, die sie als bedeutsam darstellten (Tab. 2). F56 formulierte eine Stärkung ihres Glaubens sowie eine bessere Bewältigung hinsichtlich subjektiver früherer traumatischer Erfahrungen. Hieraus resultierten ein verbessertes Selbstbewusstsein sowie Festigkeit und mehr Freiraum («Ich kann letztendlich (besser) mich selber behaupten und meine Bedürfnisse erfüllen.»). F63 erwähnte, dass sie Dinge nicht mehr so ernst nehme wie zuvor. Sie könne besser zwischen wichtigen und weniger wichtigen Dingen unterscheiden und sage, wenn notwendig, entweder «ja» oder «nein». F69 fand den Mut, eine Reise zu ihrer weit entfernt lebenden Tochter zu planen, obwohl sie nicht gerne reise.

F42 setzte ihr Bedürfnis um, ihre Aktivitäten an ihren persönlichen Tagesrhythmus anzupassen, was unter anderem Auswirkungen auf die Verabreichung der konventionellen Therapien hat. Zudem äusserte diese Patientin 
bestimmte Erwartungen an das komplementärmedizinische Behandlungssetting, das sie im Zusammenhang mit dem MT-Angebot als befriedigend empfand, nach Gesprächen/hinterlegtem Menschenbild, zeitlicher Ausdehnung des Angebotes. Auch versuchte sie eine neue Gestaltung und Orientierung ihrer Ausrichtung an die Arbeit und berichtete über ein Gefühl der Befreiung ( $« . .$. ich merke jetzt auch in Bezug auf Beziehungen, ... ich muss jetzt nicht mehr überall dabei sein. Ich darf mich abgrenzen. Für mich ist das fast etwas wie eine kleine Erleichterung.»).

\section{Diskussion}

Eine gelungene Krankheitsbewältigung kann sich - ganzheitlich betrachtet und vor dem Hintergrund eines konkret aufgefassten biopsychosozialen Modells - nicht nur auf quantitative Parameter wie die Verlängerung der Lebenszeit beziehen. Gleichermassen genügt hinsichtlich einer lebensqualitativen Betrachtung nicht, diese auf eine funktionale bzw. symptomatische Verbesserung zu beschränken. Eine gelungene Krankheitsbewältigung umfasst insofern eine motivational-sinnhaft hinterlegte Abgleichung der Behandlungsstrategie in Bezug auf das subjektive Krankheitsparadigma. Die Wahl einzelner therapeutischer Bausteine befriedigt hierbei «Bedürfnisse», die sich an subjektiv-kausalen ätiologischen Faktoren, Deutungen sowie individuellen Vorstellungen orientieren hinsichtlich der Bedeutung der Erkrankung und der Bewertung therapeutischer Methoden/Settinganforderungen inklusive der subjektiv notwendigen, fachlich begleitenden Unterstützung. Dies würde in der Konsequenz bedeuten, dass das Gefühl einer individuell guten QoL auch davon abhängt, dass man nicht nur punktuell das richtige Mittel nimmt, sondern kontextuell das Angemessene/Mögliche und damit subjektiv Richtige unternimmt.

Bezüglich der verschiedenen Behandlungsmethoden in einem multimodalen Konzept können mannigfaltige Modellvorstellungen angesprochen werden, wie z.B. Chemotherapie/Operation/Bestrahlung versus MT, «Naturheilmittel» mit «alternativem» Medizinalparadigma sowie assoziierter Beziehungsgestaltung/Behandlungssetting und damit verbundenen «Ritualen» wie sich wiederholenden Injektionen und Selbstinjektionen. Sodann betrifft die Wahl weiterer therapeutischer Bausteine das Thema der Selbststeuerung (Autonomie), was im Rahmen einer psychosomatischen Betrachtung relevant erscheint.

Unter der laufenden Behandlung nahmen die Patientinnen an sich selbst verschiedene Veränderungen ihre QoL betreffend wahr - z.B. im Zusammenhang mit einer höheren Vitalität, einer verbesserten Autonomie sowie gesteigerten persönlichen Leistungen.

Unsere Beobachtungen zeigten entsprechende $\mathrm{Zu}$ sammenhänge eindrücklich auf: Auch wenn eine von den oben genannten Patientinnen (F42) beispielsweise «weiss», dass ihre subjektive Selbstvernachlässigung nicht die eigentliche Ursache ihrer Erkrankung ist, so kann in der Umkehr eine «neu» mehr gelebte selbstfürsorgliche Orientierung (Selbstermächtigung, Stärkung der Selbstwirksamkeit) einen wichtigen Impuls zur Stabilisierung der Hoffnung, eines wichtigen Lebensqualitätsparameters, darstellen. Folgerichtig äusserte diese Patientin hinsichtlich der MT, dass sie - solange die Krankheit noch nicht abschliessend behandelt sei - davon profitiere, dass sie da noch in das Spital für die MT kommen könne, während dagegen die Chemotherapie abgeschlossen sei. Weiterführend umfasste die subjektive Krankheitsbewältigung der Patientin eine gestaltende Anpassung ihres Beziehungsumfeldes sowie eine berufliche Neuorientierung - um aus möglichen krankheitsunterhaltenden Einflussfaktoren herauszukommen. Eine weitere Patientin (F63) mochte sich zwar nicht mit vermuteten, tiefer liegenden subjektivätiologischen Komponenten auseinandersetzen - hierbei profitierte sie allerdings von «sekundären», der MT zugeordneten Wirkungen, wie stabilere Stimmung und abnehmender Schmerz, bei primär naturheilkundlicher Behandlungsstrategie. Bei der dritten Patientin (F69) implizierte die MT einen konkret unterstützenderen Miteinbezug ihrer Tochter, wobei sie sich mit deren konzeptionellen Behandlungsvorstellungen auseinandersetzen musste. Die vierte Patientin (F42) wiederum assoziierte sowohl ihren Krebs als auch gewisse Behandlungsmethoden mit krank machender Gewalt (Trauma, Behinderung ihrer Selbstentwicklung) und wählte einen primär psychotherapeutischen Behandlungszugang bzw. Methoden, die sie dabei unterstützten, in ihre Kraft zu kommen.

MT scheint - nebst konkreten lebensqualitativen Wirkungen - ein gutes Potenzial für eine integrativere (und aktivere) Bewältigung von Krebserkrankungen anzubieten, was in einer subjektiv verbesserten QoL zum Ausdruck kommt. Denkbar sind hierbei beispielsweise «indirekte» Wirkungen wie eine gestärkte Hoffnung über eine z.B. verbesserte subjektiv-sinnhafte Motivierung durch den Versuch, individuelle Bedürfnisse, Vorstellungen und Handlungen - inklusive der sie unterstützenden Angehörigen - in einen Zusammenhang zu bringen. Mit Hinblick auf eine gelungene Krankheitsbewältigung muss sodann auf den Fakt hingewiesen werden, dass die Brustkrebserkrankung einige "Spezifitäten» aufweist, und zwar insofern, dass die «Brust» der Frau einen hohen Symbolcharakter hat (nährendes Prinzip; Geliebte/Mutter), einen wesentlichen Teil des weiblichen Selbstbildes darstellt und somit eine identitätsstiftende Komponente aufweist - eine operative «Sanierung» kann diesbezüglich mit einer körperlichen und seelischen Wunde assoziiert sein. Eine ganzheitliche, lebensqualitativ orientierte Krankheitsbewältigung ist vor diesem Hintergrund mit einem hohen Anspruch verbunden. 


\section{Dank}

Diese Veröffentlichung ist den PatientInnen gewidmet, die an dieser Studie teilnahmen und dadurch ihre Erfahrungen, die sie in einer sehr herausfordernden Phase ihres Lebens hatten, mit uns und den Lesern teilen. Dank schulden wir ebenso den Angehörigen der PatientInnen, die ihre Teilnahme an der Studie ermöglichten und ebenfalls an den Interviews teilnahmen, ebenso den Ärzten, die Patienten rekrutierten, speziell Dr. S. Obrist. Wir danken Dr. S. Haile (Universität Zürich, Schweiz) für statistische Beratung und Frau Isabella Herr (Abteilung für Pflegeentwicklung, Paracelsus-Spital Richterswil, Schweiz) für die Übersetzung einiger Textabschnitte in die deutsche Sprache. Diese Arbeit wurde von Weleda AG, Arlesheim, Schweiz, finanziert.

\section{Literatur}

1 Kienle G, Kiene H: Complementary cancer therapy: a systematic review of prospective clinical trials on anthroposophic mistletoe extracts. Eur J Med Res 2007;12:103-119.

2 Molassiotis A, Fernadez-Ortega P, Pud D, Ozden G, Scott JA, Panteli V, Margulies A, Browall M, Magri M, Selvekerova S, Madsen E, Milovics L, Bruyns I, Gudmundsdottir G, Hummerston S, Ahmad AM, Platin N, Kearney N, Patiraki E: Use of complementary and alternative medicine in cancer patients: a European survey. Ann Oncol 2005;16:655663.

3 Ostermann T, Raak C, Büssing A: Survival of cancer patients treated with mistletoe extract (Iscador): a systematic literature review. BMC Cancer 2009;9:451.

\section{Disclosure Statement}

In den letzten Jahren bekamen APS-W und LR gelegentlich finanzielle Unterstützung von Mistelherstellern.
4 Melzer J, Iten F, Hostanska K, Saller R: Efficacy and safety of mistletoe preparations (Viscum album) for patients with cancer diseases. A systematic review. Forsch Komplementmed 2009;16:217-226.

5 Kienle GS, Glockmann A, Schink M, Kiene H: Viscum album L. extracts in breast and gynaecological cancers: a systematic review of clinical and preclinical research. J Exp Clin Cancer Res 2009;28:79.

6 Horneber MA, Bueschel G, Huber R, Linde $\mathrm{K}$, Rostock M: Mistletoe therapy in oncology. Cochrane Database Syst Rev 2008;2:CD003297.

7 Kienle GS, Kiene H: Review article: Influence of Viscum album L (European mistletoe) extracts on quality of life in cancer patients: a systematic review of controlled clinical studies. Integr Cancer Ther 2010;9:142-157.
8 Brandenberger M, Simões-Wüst AP, Rostock M, Rist L, Saller R: An exploratory study on the quality of life and individual coping of cancer patients during mistletoe therapy. Integr Cancer Ther 2011;DOI: $10.1177 / 1534735411413267$.

9 Mayring P: Einführung in die qualitative Sozialforschung. Eine Anleitung zu qualitativem Denken, ed 3. Weinheim, Psychologie Verlags Union, 1996.

10 Gläser J, Laudel G: Experteninterviews und qualitative Inhaltsanalyse, ed 3. Wiesbaden, Verlag für Sozialwissenschaften, 2009, p 347.

11 Brandenberger M: Untersuchung zur Lebensqualität (QoL) unter Misteltherapie bei Krebspatienten mit polymodalem Therapiekonzept. Zürich, Medical Faculty, University of Zurich, 2010, p 262. 